\title{
An Evaluation of the Potential of Cytochrome P450 3A4-Mediated Drug- Drug Interactions with Desvenlafaxine Use
}

\author{
Alice Nichols ${ }^{1 *}$, Yali Liang ${ }^{1}$, Kyle Matschke ${ }^{1}$, Jeff Paul ${ }^{2}$, Jessica Behrle ${ }^{2}$, Joel Posener ${ }^{2}$, Alain Patat $^{2}$, Shannon Lubaczewski ${ }^{1}$, Gabriel \\ Braley ${ }^{1}$ and Tanya Ramey ${ }^{3}$
}

${ }^{1}$ Clinical Pharmacology, Primary Care, Pfizer Inc., 500 Arcola Drive, Collegeville, PA 19426, USA

${ }^{2}$ Formerly of Pfizer Inc, Collegeville, PA, USA

${ }^{3}$ Pfizer Inc, Groton, CT, USA

\begin{abstract}
A series of 3 open-label, 2-period, sequential studies were conducted to assess the impact of desvenlafaxine on cytochrome P450 3A4 (CYP3A4) enzyme-mediated metabolism, and the effect of CYP3A4 inhibition on desvenlafaxine metabolism. Study 1 evaluated a single dose of desvenlafaxine $400 \mathrm{mg}$ administered alone or with a CYP3A4 inhibitor (ketoconazole [ $400 \mathrm{mg} / \mathrm{d}$ for 8 days]). Studies 2 and 3 evaluated a single dose of CYP3A4 substrate (midazolam [4 mg]) administered alone or with multiple doses of desvenlafaxine $400 \mathrm{mg}$ and $50 \mathrm{mg}$ (the recommended therapeutic dose), respectively, to assess the potential inhibitory effect of desvenlafaxine. In study 1 , desvenlafaxine peak plasma concentration $\left(\mathrm{C}_{\max }\right)$ and area under the plasma concentration-versus-time curve (AUC) geometric least-squares mean ratios (desvenlafaxine and ketoconazole vs. desvenlafaxine alone) were $108 \%(90 \%$ confidence interval [Cl], $100 \%$ to $117 \%)$ and $143 \%(90 \% \mathrm{Cl}, 138 \%$ to $149 \%)$, respectively. Total oral clearance decreased by $29 \%$ with ketoconazole coadministration. In studies 2 and $3, C_{\max }$ and AUC geometric least-squares mean ratios (midazolam and desvenlafaxine vs. midazolam alone) were $84 \%(90 \% \mathrm{Cl}, 72 \%$ to $97 \%)$ and $69 \%(90 \%$ $\mathrm{Cl}, 61 \%$ to $78 \%)$, respectively, for the desvenlafaxine $400-\mathrm{mg}$ dose, and $86 \%(90 \% \mathrm{Cl}, 79 \%$ to $94 \%)$ and $71 \%(90 \%$ $\mathrm{Cl}, 65 \%$ to $78 \%$ ), respectively, for the desvenlafaxine $50-\mathrm{mg}$ dose. No serious adverse events or safety-related discontinuations occurred. Desvenlafaxine is minimally metabolized by CYP3A4 and does not appear to inhibit CYP3A4.
\end{abstract}

Keywords: Desvenlafaxine; Ketoconazole; Midazolam; Cytochrome P450 3A4; Drug-drug interactions

Abbreviations: AEs: Adverse Events; AUC: Area Under the Plasma Concentration-Versus-Time Curve from Time Zero to Infinity; $\mathrm{AUC}_{\mathrm{T}}$ : AUC from Time Zero to the Last Observed Plasma Concentration at Time T; BMI: Body Mass Index; CI: Confidence Interval; $\mathrm{Cl} / \mathrm{F}$ : Apparent Oral-dose Clearance; $\mathrm{CL}_{\mathrm{R}}$ : Renal Clearance; $\mathrm{C}_{\max }$ : Peak Plasma Concentration; CYP3A4: Cytochrome P450 3A4; GLSM: Geometric Least-squares Mean; TEAEs: Treatment-emergent AEs; $\mathrm{t}_{\max }$ : Time to $\mathrm{C}_{\max } ; \mathrm{V}_{\mathrm{z}} / \mathrm{F}$ : Apparent Volume of Distribution

\section{Introduction}

Desvenlafaxine (administered as desvenlafaxine succinate) is a serotonin-norepinephrine reuptake inhibitor approved by the US Food and Drug Administration for the once-daily treatment of major depressive disorder [1]. Desvenlafaxine is metabolized primarily by phase II enzymes to form the glucuronide conjugate and to a lesser extent by the phase I enzyme cytochrome P450 3A4 (CYP3A4) to form $\mathrm{N}, \mathrm{O}$-didesmethylvenlafaxine $[1,2]$. Because CYP3A4 metabolism is a minor metabolic pathway for desvenlafaxine, it is relevant to assess the risk of interactions involving drugs mediated by this enzyme.

The primary objectives of the 3 studies reported here were (1) to evaluate the effect of ketoconazole, an established CYP3A4 inhibitor [3], on the pharmacokinetics and safety of desvenlafaxine when coadministered with ketoconazole in healthy young subjects (study 1); (2) to assess the potential inhibitory effect of desvenlafaxine when coadministered with midazolam, an established substrate of CYP3A4 [3], on the pharmacokinetics of midazolam and 1'-hydroxymidazolam (the primary active metabolite of midazolam, produced via CYP3A4 metabolism [4,5]) (studies 2 and 3); and (3) to evaluate the safety of coadministered desvenlafaxine and midazolam in healthy young subjects (studies 2 and 3 ). A secondary objective of study 3 was to describe the multiple-dose pharmacokinetics of the recommended therapeutic dose of desvenlafaxine $50 \mathrm{mg}$, for which only single-dose pharmacokinetic data are currently available.

\section{Methods}

\section{Subjects}

All 3 studies enrolled men or nonlactating, nonpregnant adult women aged 18 to 45 years (studies 1 and 2) or 18 to 55 years (study 3) with a body mass index (BMI) of 18 to $30 \mathrm{~kg} / \mathrm{m}^{2}$ (studies 1 and 2) or 17.5 to $30.5 \mathrm{~kg} / \mathrm{m}^{2}$ (study 3) who were healthy, as determined by the investigator. Subjects were nonsmokers or smoked fewer than 10 cigarettes per day.

Subjects were excluded from participation if they had significant cardiovascular, hepatic, renal, respiratory, gastrointestinal, endocrine, immunologic, dermatologic, hematologic, neurologic, or psychiatric disease; a positive orthostatic test result at screening; a history of seizure disorder; glaucoma or increased intraocular pressure (studies 2 and 3 ); a condition that could interfere with the absorption, distribution,

*Corresponding author: Alice Nichols, Senior Director, Clinical Pharmacology Primary Care, Pfizer Inc., 500 Arcola Drive, Collegeville, PA 19426, USA, Tel: 484865-8741; Fax: 484-865-9075; E-mail: alice.nichols@pfizer.com

Received December 20, 2012; Accepted January 21, 2013; Published January 27, 2013

Citation: Nichols A, Liang Y, Matschke K, Paul J, Behrle J, et al. (2013) An Evaluation of the Potential of Cytochrome P450 3A4-Mediated Drug-Drug Interactions with Desvenlafaxine Use. J Bioequiv Availab 5: 053-059. doi:10.4172/ jbb.1000134

Copyright: ( 2013 Nichols A, et al. This is an open-access article distributed under the terms of the Creative Commons Attribution License, which permits unrestricted use, distribution, and reproduction in any medium, provided the original author and source are credited. 
metabolism, or excretion of study drugs; acute disease (eg, nausea, vomiting, fever, diarrhea) within 7 days of study day 1 (studies 1 and 3) or day -1 (study 2); alcohol or drug abuse; positive serologic findings for human immunodeficiency virus antibodies, hepatitis B surface antigen, or hepatitis $\mathrm{C}$ virus antibodies; allergy to ketoconazole (study 1), midazolam or other benzodiazepines (studies 2 and 3), cherries (study 2), desvenlafaxine, or venlafaxine; donation of blood within 60 days (study 1) or 56 days (studies 2 and 3); use of any investigational or prescription drug within 30 days (studies 1 and 2), prescription drug within 7 days (study 3), or St. John's wort (studies 1 and 2) within 30 days, over-the-counter drugs within 7 days (study 3 ) or 14 days (studies 1 and 2), or herbal supplements within 28 days (study 3) or 14 days (studies 1 and 2) (excluding acetaminophen or vitamins), and tobacco or caffeine within 24 hours (study 3 ) or 48 hours before study day 1 . Subjects who consumed grapefruit or grapefruit-related citrus fruits within 7 days before the first dose of study medication were excluded from study 3 .

\section{Study design and procedures}

Each of the 3 studies was an open-label, nonrandomized, 2-period, sequential, inpatient study conducted in healthy subjects at a single investigational site (study 1: Rueil Malmaison, France; study 2: Evansville, IN; study 3: New Haven, CT) in accordance with ethical principles that have origins in the Declaration of Helsinki and any amendments in place when the study was conducted. The studies were designed and performed in compliance with Good Clinical Practice. The protocols and informed consent forms were submitted to an independent ethics committee for review and written approval.

In study 1 , a single dose of desvenlafaxine $(400 \mathrm{mg})$ was administered alone. After a 4-day washout, subjects received the CYP3A4 inhibitor ketoconazole (200 mg twice daily) for 8 consecutive days; concomitant desvenlafaxine ( $400 \mathrm{mg}$ ) was given with the morning dose of ketoconazole on day 4. Ketoconazole administration continued throughout the remaining 96 hours, during which blood and urine samples were collected for pharmacokinetic analysis. In study 2 , a single 4-mg dose of the CYP3A4 substrate midazolam was administered alone, followed by no medication for 1 day and desvenlafaxine (titrated from 100 to $400 \mathrm{mg} / \mathrm{d}$ by the fourth dose) for 12 days; concomitant midazolam $(4 \mathrm{mg})$ was given on day 8 . The desvenlafaxine dosage was then titrated downward (from $400 \mathrm{mg} / \mathrm{d}$ on day 8 to a final dose of $100 \mathrm{mg} / \mathrm{d} 3$ days later). In study 3 , a single dose of midazolam (4 $\mathrm{mg}$ ) was administered alone, followed by no medication for 1 day and desvenlafaxine ( $50 \mathrm{mg}$ once daily) for 6 days; concomitant midazolam $(4 \mathrm{mg}$ ) was given on day 6. Desvenlafaxine $200 \mathrm{mg}, 100 \mathrm{mg}$, and 50 mg tablets were supplied by Wyeth Research (Collegeville, PA, USA), and midazolam $2 \mathrm{mg} / \mathrm{mL}$ oral liquid was supplied by Wyeth Research or procured (commercially) by the clinical research unit. Ketoconazole $200 \mathrm{mg}$ tablets (Nizoral, Janssen Pharmaceutical, Titusville, NJ, USA) were supplied by the study site.

In study 1, blood samples were collected to determine the pharmacokinetics of desvenlafaxine in the absence and presence of ketoconazole. Baseline $5 \mathrm{~mL}$ blood samples were collected on days 1 and 9 before desvenlafaxine administration and at $0.5,1,2,4,6$, $8,10,12,16,24,36,48,60,72$, and 96 hours after administration. A baseline blood sample for ketoconazole was collected on day 5 before ketoconazole administration, and trough samples were collected before morning doses of ketoconazole on days 7, 8, and 9. Urine samples were collected for desvenlafaxine pharmacokinetic analysis on days 1 and 9 before desvenlafaxine administration and from 0 to 96 hours after administration.
In studies 2 and 3, blood samples were collected to determine the pharmacokinetics of midazolam in the absence and presence of desvenlafaxine. To determine midazolam and 1'-hydroxymidazolam plasma concentrations, a baseline $5 \mathrm{~mL}$ blood sample was collected on day 1 within 2 hours before midazolam administration and at $0.25,0.5$, $1,1.5,2,3,4,5,6,8,10,12,16$, and 24 hours after administration, and on day 6 (study 3 ) or day 8 (study 2), during steady-state administration of desvenlafaxine, within 2 hours before midazolam dosing and at 0.25 , $0.5,1,1.5,2,3,4,5,6,8,10,12,16$, and 24 hours after administration. In study 3, 4-mL blood samples were collected to determine desvenlafaxine plasma concentrations on day 5 within 2 hours before desvenlafaxine dosing and at $0.5,1,2,3,4,6,8,12$, and 16 hours after administration.

\section{Assays}

A validated liquid chromatography mass spectroscopy method with mass spectroscopy detection was used in study 1 to analyze plasma samples for ketoconazole and desvenlafaxine and to analyze urine samples for unconjugated and total (unconjugated plus conjugated) desvenlafaxine. The liquid chromatography mass spectroscopy method with mass spectroscopy detection also was used in study 2 to analyze plasma samples for midazolam, and 1'-hydroxymidazolam, and in study 3 to analyze plasma samples for midazolam, 1'-hydroxymidazolam, and desvenlafaxine.

\section{Safety analyses}

Safety was evaluated from spontaneously reported signs and symptoms, physical examinations (including vital sign measurements), 12-lead electrocardiograms, and clinical laboratory evaluations. All adverse events (AEs) were recorded.

\section{Pharmacokinetic analyses}

A non compartmental, model-independent method was used to analyze the plasma concentrations of desvenlafaxine, midazolam, and 1'-hydroxymidazolam using WinNonlin Professional Version 4.1 software (Pharsight Corporation, Mountain View, CA, USA) for studies 1 and 2, and an internal Pfizer software package for study 3 . All values are reported as geometric means unless otherwise indicated. Pharmacokinetic parameters included peak plasma concentration $\left(\mathrm{C}_{\max }\right)$, time to $\mathrm{C}_{\max }\left(\mathrm{t}_{\max }\right)$, area under the plasma concentration-versustime curve from time zero to infinity (AUC), area under the plasma concentration-versus-time curve from time zero to the last observed plasma concentration at time $\mathrm{T}\left(\mathrm{AUC}_{\mathrm{T}}\right)$, terminal elimination half-life $\left(\mathrm{t}_{1 / 2}\right)$, apparent oral-dose clearance $(\mathrm{Cl} / \mathrm{F}$; studies 1 and 2$)$, apparent volume of distribution $\left(\mathrm{V}_{z} / \mathrm{F}\right.$; studies 1 and 2$)$, and renal clearance $\left(\mathrm{CL}_{\mathrm{R}}\right.$; study 1).

\section{Statistical analyses}

The plasma concentration data and pharmacokinetic parameters of the substrate drug (desvenlafaxine for study 1 and midazolam for studies 2 and 3 ) were compared between monotherapy (desvenlafaxine for study 1 and midazolam for studies 2 and 3 ) and combination therapy (desvenlafaxine plus ketoconazole for study 1 and midazolam plus desvenlafaxine for studies 2 and 3 ) by using a 1 -factor analysis of variance. The magnitude of effect of the test drug with known (ketoconazole for study 1) or tested (desvenlafaxine for studies 2 and 3) inhibition for CYP3A4 on the pharmacokinetic profile of the substrate drug was estimated by calculating the geometric least-squares mean (GLSM) relative bioavailability of $\mathrm{C}_{\max }$ and AUC and $90 \%$ CI limits for the substrate drug. 


\section{Results}

\section{Study population}

Fifteen subjects (14 male; 1 female) with an average age of 32.9 years and an average BMI of $24.3 \mathrm{~kg} / \mathrm{m}^{2}$ were enrolled in and completed study 1. Thirteen subjects were Caucasian, 1 was African American, and 1 was West Indian.

A total of 26 male subjects with a mean age of 34.9 years and an average BMI of $25.2 \mathrm{~kg} / \mathrm{m}^{2}$ enrolled in study 2. Eighteen subjects were Caucasian, 7 were African American, and 1 was West Indian. Two subjects discontinued from study 2 (1 withdrew consent during titration to steady state, and 1 was withdrawn by the sponsor on day 2 because of a syringe malfunction during midazolam administration).

Study 3 enrolled 28 subjects with a mean age of 36.6 years and a mean BMI of $26.0 \mathrm{~kg} / \mathrm{m}^{2}$ (women) and $26.5 \mathrm{~kg} / \mathrm{m}^{2}$ (men). Eighteen subjects were African American, 6 were Caucasian, and 4 were of other races. Data from 2 subjects were removed from the pharmacokinetic statistics of desvenlafaxine and midazolam and statistical comparisons of midazolam pharmacokinetics with and without concomitant administration of desvenlafaxine because of extremely low to nonquantifiable desvenlafaxine concentrations on day 5 of dosing with desvenlafaxine $50 \mathrm{mg} / \mathrm{d}$.

\section{Pharmacokinetics}

In study 1 , the plasma concentration-versus-time curves for desvenlafaxine are shown in Figure 1. The GLSM ratios for $\mathrm{C}_{\max }$ and AUC of desvenlafaxine for the comparison of desvenlafaxine and ketoconazole coadministration with desvenlafaxine alone were $108 \%$ (90\% CI, $100 \%$ to $117 \%)$ and $143 \%$ (90\% CI, $138 \%$ to $149 \%)$, respectively. The $90 \%$ CIs for the ratios of the mean AUC were outside the acceptable limits of $80 \%$ to $125 \%$, which define bioequivalence [6,7]. The mean $\mathrm{Cl} / \mathrm{F}$ for desvenlafaxine was $29 \%$ lower during coadministration, but the mean $\mathrm{V}_{z} / \mathrm{F}$ was similar for both administrations (Table 1). The mean $t_{1 / 2}$ was approximately $38 \%$ longer and the $t_{\max }$ was approximately $12 \%$ longer after coadministration than when desvenlafaxine was given alone.

The relative proportion of renal to total clearance of desvenlafaxine was approximately similar with only a minor increase with coadministration of ketoconazole. The geometric mean (range) ratio of $\mathrm{CL}_{\mathrm{R}}$ to $\mathrm{CL} / \mathrm{F}$ was $44 \%$ (range, $26 \%$ to $68 \%$ ) of the total clearance without coadministration and $51 \%$ (range, $37 \%$ to $67 \%$ ) with coadministration of ketoconazole. The geometric mean urinary recovery of unconjugated and total (unconjugated+conjugated) desvenlafaxine in subjects receiving desvenlafaxine alone was $44 \%$ and $61 \%$ of the $400-\mathrm{mg}$ desvenlafaxine dose, respectively (Table 1). A minor increase in urinary recoveries of unconjugated (50\%) and total desvenlafaxine (68\%) were observed when desvenlafaxine was administered with multiple doses of ketoconazole.

In study 2, the plasma concentration-versus-time curves for midazolam and 1'-hydroxymidazolam are shown in Figure 2. The GLSM ratios (midazolam and desvenlafaxine coadministration $v s$. midazolam alone) for $\mathrm{C}_{\max }$ and AUC of midazolam were $84 \%$ (90\% CI, $72 \%$ to $97 \%$ ) and $69 \%$ (90\% CI, $61 \%$ to $78 \%)$, respectively. For 1'-hydroxymidazolam, the GLSM ratios (midazolam and desvenlafaxine coadministration $v s$. midazolam alone) for $\mathrm{C}_{\max }$ and AUC were $98 \%$ (90\% CI, $85 \%$ to $113 \%$ ) and $92 \%$ (90\% CI, $87 \%$ to $98 \%$ ), respectively. The $90 \%$ CIs for $\mathrm{C}_{\max }$ and AUC for midazolam were outside the acceptable limits of $80 \%$ to $125 \%$ for bioequivalence, but for 1'-hydroxymidazolam the $90 \%$ CIs were within the acceptable range. After midazolam was coadministered with desvenlafaxine, the mean $\mathrm{Cl} / \mathrm{F}$ for midazolam was $44 \%$ higher, the mean $\mathrm{V}_{\mathrm{z}} / \mathrm{F}$ was $24 \%$ higher, the mean $\mathrm{t}_{1 / 2}$ was $12 \%$ shorter, and the median $\mathrm{t}_{\max }$ values were similar (Table 2).

In study 3, consistent with steady-state levels of desvenlafaxine

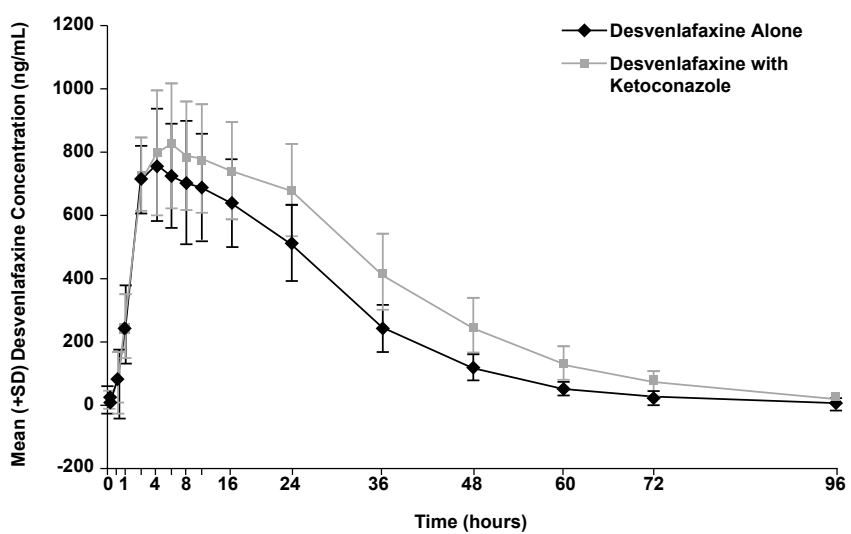

Figure 1: Mean (SD) plasma concentration-time profiles of desvenlafaxine after single oral dose of desvenlafaxine $400 \mathrm{mg} / \mathrm{d}$ with and without ketoconazole 200 $\mathrm{mg}$ twice daily in healthy subjects in study 1 .
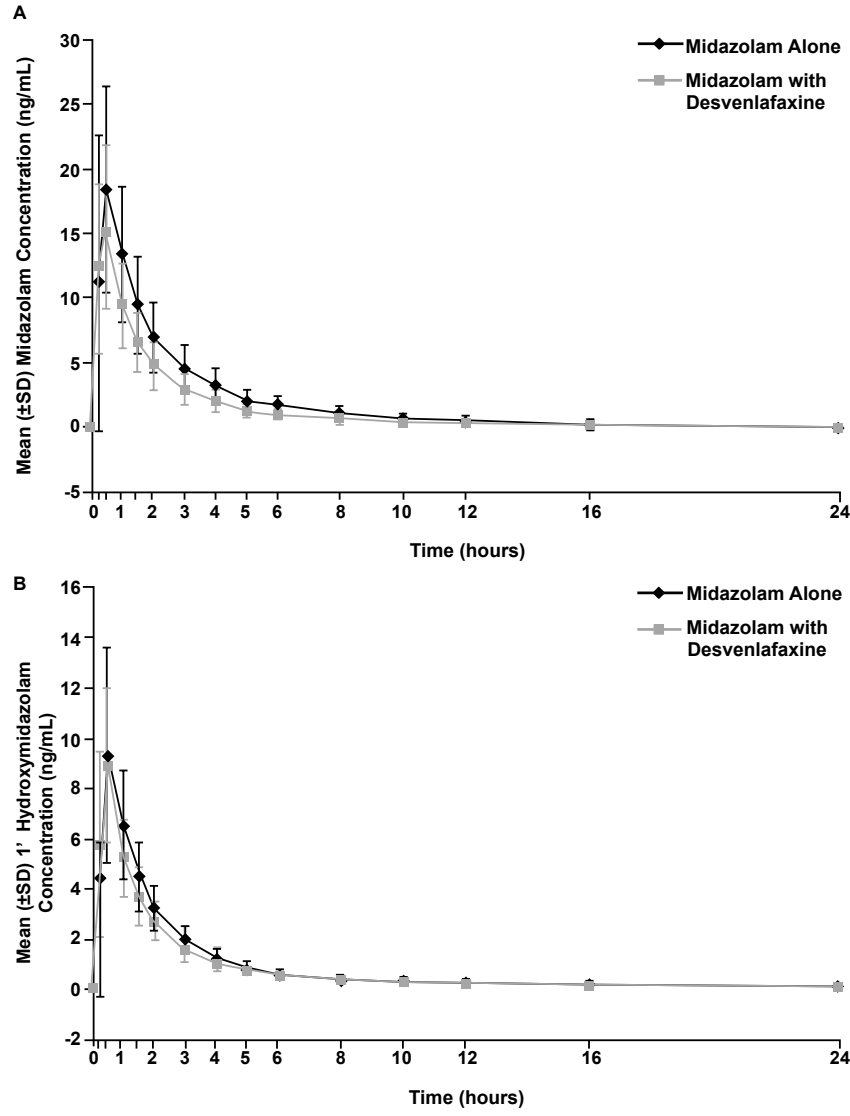

Figure 2: Mean (SD) plasma concentrations of (A) midazolam and (B) 1 '-hydroxymidazolam after single oral dose of midazolam $4 \mathrm{mg}$ alone or with desvenlafaxine $400 \mathrm{mg} / \mathrm{d}$ in healthy subjects in study 2 . 
Citation: Nichols A, Liang Y, Matschke K, Paul J, Behrle J, et al. (2013) An Evaluation of the Potential of Cytochrome P450 3A4-Mediated Drug-Drug Interactions with Desvenlafaxine Use. J Bioequiv Availab 5: 053-059. doi:10.4172/jbb.1000134

being reached by day 5 of daily dosing, the mean predose concentration (range) of desvenlafaxine on day 4 was $80 \mathrm{ng} / \mathrm{mL}(23-144 \mathrm{ng} / \mathrm{mL})$ and on day 5 was $87 \mathrm{ng} / \mathrm{mL}(35-167 \mathrm{ng} / \mathrm{mL}$ ) (Figure 3). The geometric mean for AUC from time 0 to 24 hours post-dose was 2853 (geometric $\%$ coefficient of variation: 31 ) ng.h/mL, $\mathrm{C}_{\text {max }}$ was 174.0 (geometric $\%$ coefficient of variation: 31$) \mathrm{ng} / \mathrm{mL}$, and the median time for the $t_{\max }$ was 4.00 (range: $2.00-8.00$ ) hours.

The plasma concentration-versus-time curves for midazolam and $1^{\prime}$-hydroxymidazolam for study 3 are shown in Figure 4 . The GLSM ratios for midazolam/desvenlafaxine coadministration versus midazolam alone for $\mathrm{C}_{\max }$ and AUC of midazolam were $86 \%(90 \%$ CI, $79 \%$ to $94 \%$ ) and $71 \%$ (65\% to $78 \%$ ), respectively. The $90 \%$ CIs for both parameters fell outside the $80 \%$ to $125 \%$ acceptance range for bioequivalence. Coadministration of midazolam with desvenlafaxine $50 \mathrm{mg} / \mathrm{d}$ did not change exposure to $1^{\prime}$-hydroxymidazolam; the GLSM ratios were $101 \%$ (93\% to $110 \%$ ) for $\mathrm{C}_{\max }$ and $93 \%$ (87\% to $98 \%$ ) for AUC. Mean $\mathrm{t}_{1 / 2}$ was $14 \%$ shorter for midazolam coadministered with desvenlafaxine compared with midazolam alone. Table 3 summarizes pharmacokinetic parameters for midazolam and 1'-hydroxymidazolam in subjects dosed with midazolam $4 \mathrm{mg}$ alone and following multiple doses of desvenlafaxine $50 \mathrm{mg} / \mathrm{d}$.

\section{Safety results}

In study 1 , all 15 subjects reported 1 or more treatment-emergent

\begin{tabular}{|c|c|c|c|c|c|c|c|c|c|c|}
\hline & $C_{\text {max }}, g / m L$ & $t_{\max }, h^{a}$ & $\mathrm{AUC}_{\mathrm{T}}, \mathrm{ng} \cdot \mathrm{h} / \mathrm{mL}$ & AUC, ng.h/mL & $t_{1 / 2}, h$ & CI/F, L/h/kg & $\mathrm{V}_{\mathrm{z}} / \mathrm{F}, \mathrm{L} / \mathrm{kg}$ & $\mathrm{CL}_{\mathrm{R}}, \mathrm{L} / \mathrm{h} / \mathbf{k g}$ & Recovery, unconj. (\%) & Recovery, total (\%) \\
\hline \multicolumn{11}{|c|}{ Desvenlafaxine $400 \mathrm{mg}$} \\
\hline Mean (SD) & 819 (161) & 6.00 & 21784 (4310) & 21942 (4321) & $9.79(0.98)$ & $0.26(0.05)$ & $3.59(0.63)$ & $\begin{array}{l}0.117 \\
(0.035)\end{array}$ & $46(13)$ & $63(15)$ \\
\hline$\% \mathrm{CV}$ & 19.7 & & 19.8 & 19.7 & 10.0 & 17.8 & 17.6 & 30.0 & 27.5 & 23.3 \\
\hline $\begin{array}{l}\text { Geometric } \\
\text { mean }\end{array}$ & 804 & & 21398 & 21557 & 9.75 & 0.25 & 3.54 & 0.111 & 44 & 61 \\
\hline Min, Max & 562,1105 & $4.00,16.03$ & 15568,31485 & 15607,31654 & $8.14,11.36$ & $0.17,0.33$ & $2.71,4.78$ & $\begin{array}{l}0.049 \\
0.165\end{array}$ & 25,68 & 34,78 \\
\hline \multicolumn{11}{|c|}{ Desvenlafaxine $400 \mathrm{mg}+$ ketoconazole $200 \mathrm{mg}$} \\
\hline Mean (SD) & $884(185)$ & 8.00 & $30820(6260)$ & $31245(6463)$ & $13.49(1.47)$ & $0.18(0.03)$ & $3.50(0.60)$ & $\begin{array}{l}0.092 \\
(0.017)\end{array}$ & $51(8)$ & $68(9)$ \\
\hline$\% \mathrm{CV}$ & 21.0 & & 20.3 & 20.7 & 10.9 & 18.3 & 17.1 & 19.0 & 15.2 & 13.1 \\
\hline $\begin{array}{l}\text { Geometric } \\
\text { mean }\end{array}$ & 865 & & 30301 & 30702 & 13.42 & 0.18 & 3.45 & 0.090 & 50 & 68 \\
\hline Min, Max & 631,1192 & $4.00,16.00$ & 23914,47640 & 24079,48697 & $10.94,16.20$ & $0.11,0.23$ & $2.32,4.85$ & $\begin{array}{l}0.061 \\
0.124\end{array}$ & 37,65 & 44,77 \\
\hline
\end{tabular}

$\mathrm{CV}$ : coefficient of variation; Max: maximum; Min: minimum

${ }^{a}$ Median data are presented for $\mathrm{t}_{\max }$

Table 1: Pharmacokinetic results for study 1 (desvenlafaxine).

\begin{tabular}{|c|c|c|c|c|c|c|c|}
\hline & $C_{\max }, n g / m L$ & $t_{\max }, h^{a}$ & $\mathrm{AUC}_{\mathrm{T}}, \mathrm{ng} \cdot \mathrm{h} / \mathrm{mL}$ & AUC, ng.h/mL & $t_{1 / 2}, h$ & $\mathrm{Cl} / \mathrm{F}, \mathrm{L} / \mathrm{h} / \mathbf{k g}$ & $V_{z} / F, L / k g$ \\
\hline \multicolumn{8}{|l|}{ Midazolam $4 \mathrm{mg}$} \\
\hline \multicolumn{8}{|l|}{ Midazolam } \\
\hline Mean (SD) & $20(9.02)$ & 0.50 & 44.52 (19.78) & $45.72(20.31)$ & $4.29(1.63)$ & $1.31(0.54)$ & 7.54 (3.54) \\
\hline$\% \mathrm{CV}$ & 45 & & 44 & 44 & 38 & 41 & 47 \\
\hline Geometric mean & 18.3 & & 40.78 & 41.90 & 3.96 & 1.21 & 6.89 \\
\hline Min, Max & $7.37,48.4$ & $0.25,1.00$ & $20.50,103.8$ & $21.12,106.6$ & $1.48,7.31$ & $0.49,2.30$ & $3.92,16.70$ \\
\hline \multicolumn{8}{|c|}{ 1'-Hydroxymidazolam } \\
\hline Mean (SD) & $9.73(4.32)$ & 0.50 & $18.94(6.39)$ & $19.99(6.42)$ & $4.72(2.15)$ & NA & NA \\
\hline$\% \mathrm{CV}$ & 44 & & 34 & 32 & 45 & NA & NA \\
\hline Geometric mean & 8.86 & & 17.90 & 19.02 & 4.27 & NA & NA \\
\hline Min, Max & $4.24,19.50$ & $0.25,1.00$ & $9.55,32.05$ & $10.28,33.77$ & $1.57,9.05$ & NA & NA \\
\hline \multicolumn{8}{|c|}{ Midazolam $4 \mathrm{mg}$ + desvenlafaxine $400 \mathrm{mg}$} \\
\hline \multicolumn{8}{|l|}{ Midazolam } \\
\hline Mean (SD) & $16.5(6.24)$ & 0.50 & $30.62(12.81)$ & $31.41(13.03)$ & $3.79(1.75)$ & $1.89(0.76)$ & $9.36(4.85)$ \\
\hline$\% \mathrm{CV}$ & 38 & & 42 & 41 & 46 & 40 & 52 \\
\hline Geometric mean & 15.4 & & 28.17 & 28.95 & 3.39 & 1.75 & 8.53 \\
\hline Min, Max & $6.32,29.2$ & $0.25,1.00$ & $14.20,58.72$ & $14.94,59.76$ & $1.54,6.82$ & $0.73,3.31$ & $4.55,28.40$ \\
\hline \multicolumn{8}{|c|}{ 1'-Hydroxymidazolam } \\
\hline Mean (SD) & $9.27(3.29)$ & 0.50 & $16.33(4.83)$ & $17.44(5.56)$ & $5.51(4.30)$ & NA & NA \\
\hline$\% \mathrm{CV}$ & 35 & & 30 & 32 & 78 & NA & NA \\
\hline Geometric mean & 8.72 & & 15.69 & 16.59 & 4.39 & NA & NA \\
\hline Min, Max & $4.65,16.7$ & $0.25,1.00$ & $9.54,27.69$ & $10.01,26.23$ & $1.88,15.43$ & NA & NA \\
\hline
\end{tabular}

aMedian data are presented for $t_{\text {max }}$ 


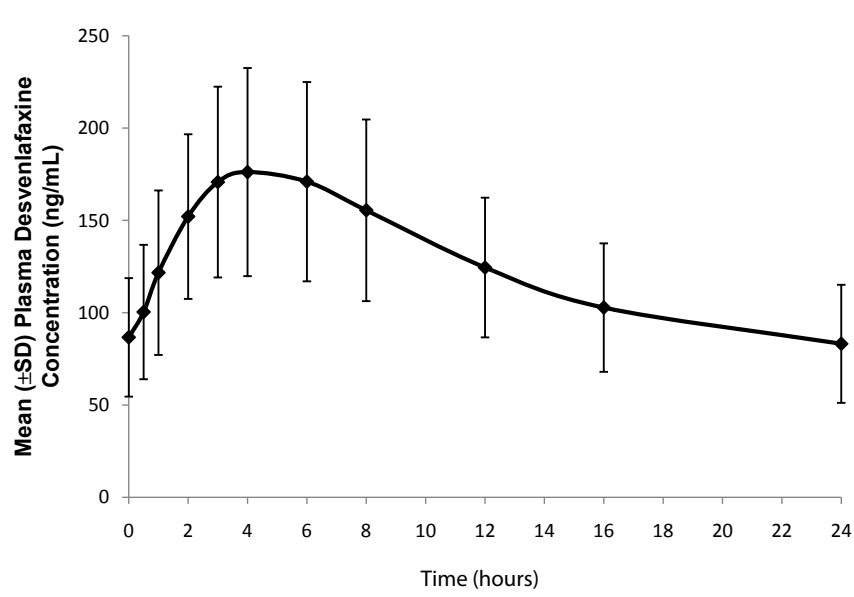

Figure 3: Mean (SD) desvenlafaxine concentration, day 5 after daily administration of desvenlafaxine $50 \mathrm{mg} / \mathrm{d}$ in healthy subjects in study 3 .

AEs (TEAEs) during the 4-day period following a single dose of desvenlafaxine (Table 4). During the 4-day treatment period in which subjects received ketoconazole, only $1(6.7 \%)$ subject reported a TEAE. All 15 subjects reported TEAEs in the 4-day period following the coadministration of ketoconazole with desvenlafaxine.

In study 2 , safety data were obtained from 26 subjects, although 2 subjects who discontinued prematurely for personal reasons did not contribute data to all study periods. Of the 26 subjects, $13(50 \%)$ reported TEAEs (Table 5). Four of 26 (15.4\%) subjects had TEAEs in the 2 days after midazolam administration, 12 of 25 (48.0\%) had TEAEs during the desvenlafaxine titration period (7 days), 2 of 24 (8.3\%) subjects had TEAEs during the 24 hours following administration of midazolam with desvenlafaxine, and 2 of 24 (8.3\%) subjects had TEAEs during desvenlafaxine taper (3 days). TEAEs were more frequent during desvenlafaxine titration than during other study periods and included headache, constipation, and asthenia, each reported by 3 of $25(12.0 \%)$ subjects.

In study 3, TEAEs were reported in 21 subjects administering midazolam $4 \mathrm{mg}, 16$ administering desvenlafaxine $50 \mathrm{mg} / \mathrm{d}$, and 24 administering desvenlafaxine $50 \mathrm{mg} / \mathrm{d}$ plus midazolam $4 \mathrm{mg}$ (Table 6). All TEAEs were mild except for 4 subjects who experienced moderate TEAEs ( 1 subject with constipation and 3 subjects with somnolence). During the initial midazolam administration and the combined desvenlafaxine and midazolam treatment periods, somnolence was the most commonly reported TEAE. In the desvenlafaxine treatment period, headache, abdominal pain, nausea, fatigue, and insomnia were the most commonly reported TEAEs.

No deaths or serious AEs occurred during any of the studies, and no subjects withdrew because of safety issues.

\section{Discussion}

Modest increases were observed in desvenlafaxine $\mathrm{C}_{\max }$ and AUC values when desvenlafaxine was coadministered with ketoconazole. When coadministered with desvenlafaxine $400 \mathrm{mg}$, minor decreases were observed in the mean $\mathrm{C}_{\max }$ and $\mathrm{AUC}$ values for midazolam (decrease of $16 \%$ and $31 \%$, respectively) and 1'-hydroxymidazolam (decrease of $2 \%$ and $8 \%$, respectively). When coadministered with desvenlafaxine $50 \mathrm{mg}$, similar decreases were observed in the mean
$\mathrm{C}_{\max }$ and AUC values for midazolam (decrease of $14 \%$ and $29 \%$, respectively) and the AUC values for its metabolite (decrease of 7\%). However, the $\mathrm{C}_{\max }$ for 1'-hydroxymidazolam increased slightly (1\%) with coadministration of desvenlafaxine $50 \mathrm{mg}$. These results indicate that desvenlafaxine is minimally metabolized by CYP3A4 with no observed inhibition of CYP3A4 metabolism of coadministered drugs. Other antidepressant agents, such as nefazodone [8] and fluoxetine [9], have been shown to inhibit CYP3A4 metabolism. Concomitant administration of agents that inhibit CYP3A4 metabolism with drugs metabolized by CYP3A4 may result in clinically important drug-drug interactions.

The increase in desvenlafaxine AUC observed during coadministration of desvenlafaxine and ketoconazole indicates an increase in oral bioavailability, which may result from decreased desvenlafaxine clearance. Although glucuronidation and renal excretion of desvenlafaxine are the primary routes of elimination, the CYP3A4-mediated metabolism of desvenlafaxine to $\mathrm{N}, \mathrm{O}$ -
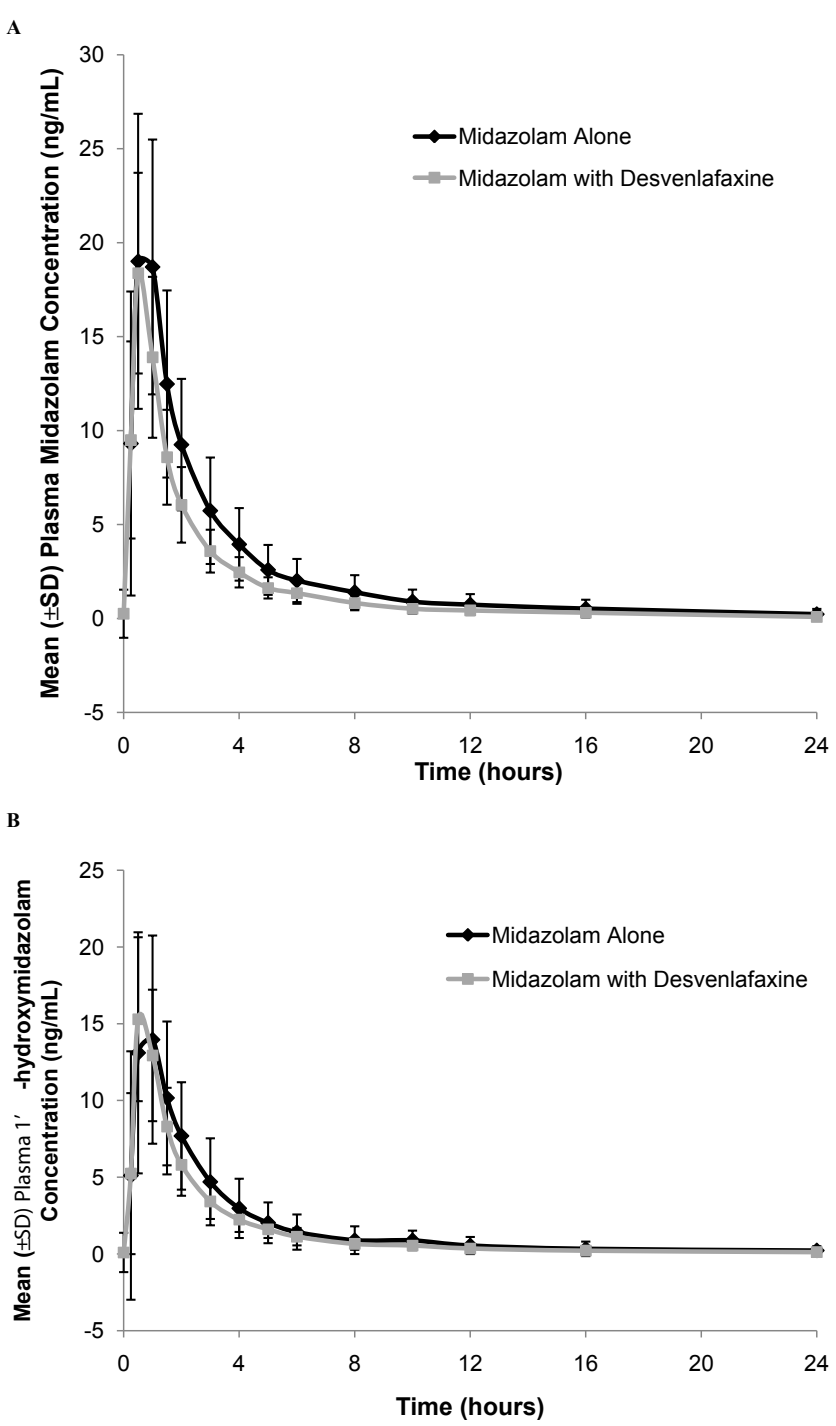

Figure 4: Mean (SD) plasma concentrations of (A) midazolam and (B) 1 '-hydroxymidazolam after single oral dose of midazolam $4 \mathrm{mg}$ alone or with desvenlafaxine $50 \mathrm{mg} / \mathrm{d}$ in healthy subjects. 
Citation: Nichols A, Liang Y, Matschke K, Paul J, Behrle J, et al. (2013) An Evaluation of the Potential of Cytochrome P450 3A4-Mediated Drug-Drug Interactions with Desvenlafaxine Use. J Bioequiv Availab 5: 053-059. doi:10.4172/jbb.1000134

\begin{tabular}{|c|c|c|c|c|c|}
\hline & $C_{\max }, n g / m L$ & $t_{\max }, h^{a}$ & $A U C_{T}$, ng.h/mL & AUC, ng.h/mL & $t_{1 / 2}, h$ \\
\hline \multicolumn{6}{|l|}{ Midazolam $4 \mathrm{mg}$} \\
\hline \multicolumn{6}{|l|}{ Midazolam } \\
\hline Mean (SD) & $22.29(7.12)$ & 0.52 & $56.50(23.98)$ & $58.77(26.67)$ & $5.32(1.59)$ \\
\hline$\% \mathrm{CV}$ & 32 & & 42 & 45 & 30 \\
\hline Geometric mean & 21.20 & & 52.85 & 54.69 & \\
\hline Min, Max & $7.86,44.3$ & $0.25,1.50$ & 26.6, 149 & $27.4,166$ & $2.55,8.62$ \\
\hline \multicolumn{6}{|c|}{ 1'-Hydroxymidazolam } \\
\hline Mean (SD) & $16.25(6.53)$ & 1.00 & $42.75(25.37)$ & $43.30(28.23)$ & $5.40(2.06)$ \\
\hline$\% \mathrm{CV}$ & 40 & & 59 & 65 & 38 \\
\hline Geometric mean & 14.93 & & 37.09 & 36.87 & \\
\hline Min, Max & $5.22,28.0$ & $0.25,1.50$ & $11.4,132$ & $12.0,138$ & $2.62,11.0$ \\
\hline \multicolumn{6}{|c|}{ Midazolam $4 \mathrm{mg}$ + desvenlafaxine $400 \mathrm{mg}$} \\
\hline \multicolumn{6}{|l|}{ Midazolam } \\
\hline Mean (SD) & $18.92(5.11)$ & 0.50 & $39.83(12.52)$ & $41.33(13.30)$ & $4.65(1.74)$ \\
\hline$\% \mathrm{CV}$ & 27 & & 31 & 32 & 38 \\
\hline Geometric mean & 18.24 & & 38.11 & 39.45 & \\
\hline Min, Max & $9.52,29.2$ & $0.25,1.02$ & $22.6,72.3$ & $23.9,73.5$ & $2.12,7.99$ \\
\hline \multicolumn{6}{|c|}{ 1'-Hydroxymidazolam } \\
\hline Mean (SD) & $16.21(6.25)$ & 0.50 & $35.24(14.88)$ & $33.43(14.65)$ & $4.62(1.80)$ \\
\hline$\% \mathrm{CV}$ & 39 & & 42 & 44 & 39 \\
\hline Geometric mean & 15.10 & & 32.42 & 30.74 & \\
\hline Min, Max & $7.27,28.7$ & $0.25,1.02$ & $14.8,68.0$ & $15.4,61.6$ & $2.66,9.89$ \\
\hline
\end{tabular}

amedian data are presented for $t_{\max }$

Table 3: Plasma pharmacokinetic results for study 3 (midazolam and 1'-hydroxymidazolam).

\begin{tabular}{|c|c|c|c|}
\hline & Desvenlafaxine $n=15 n(\%)$ & Ketoconazole $n=15 n(\%)$ & Desvenlafaxine + Ketoconazole $n=15 n(\%)$ \\
\hline Any adverse event, n (\%) & $15(100)$ & $1(6.7)$ & $15(100)$ \\
\hline Somnolence & $8(53.3)$ & 0 & $12(80.0)$ \\
\hline Insomnia & $7(46.7)$ & 0 & $6(40.0)$ \\
\hline Myadriasis & $7(46.7)$ & 0 & 8 (53.3) \\
\hline Dizziness & $6(40.0)$ & 0 & $9(60.0)$ \\
\hline Nausea & $6(40.0)$ & 0 & $11(73.3)$ \\
\hline Impotence & $3(20.0)$ & 0 & $2(13.3)$ \\
\hline Yawn & $3(20.0)$ & 0 & $2(13.3)$ \\
\hline Asthenia & $2(13.3)$ & 0 & 0 \\
\hline Headache & $1(6.7)$ & $1(6.7)$ & $5(33.3)$ \\
\hline Muscle spasms & $1(6.7)$ & 0 & $2(13.3)$ \\
\hline Tongue discoloration & $1(6.7)$ & 0 & $3(20.0)$ \\
\hline Vasodilatation & $1(6.7)$ & 0 & $3(20.0)$ \\
\hline Vomiting & $1(6.7)$ & 0 & $2(13.3)$ \\
\hline Dry mouth & 0 & 0 & $4(26.7)$ \\
\hline Nervousness & 0 & 0 & $3(20.0)$ \\
\hline Tremor & 0 & 0 & $3(20.0)$ \\
\hline
\end{tabular}

Table 4: Treatment-emergent adverse events observed in $\geq 2$ subjects during study 1 .

didesmethylvenlafaxine represents a minor metabolic pathway that is expected to be inhibited in the presence of a CYP3A4 inhibitor such as ketoconazole [2]. Therefore, the CYP3A4 inhibition of the metabolism of desvenlafaxine to $\mathrm{N}, \mathrm{O}$-didesmethylvenlafaxine could be a reasonable explanation for the observed mild increase in the desvenlafaxine AUC.

Midazolam concentrations are expected to increase in the presence of CYP3A4 inhibitors because midazolam is primarily eliminated by CYP3A4-mediated metabolism to 1'-hydroxymidazolam. Accordingly, concentrations of 1'-hydroxymidazolam are expected to decrease. Because the AUCs for midazolam and 1'-hydroxymidazolam both decreased, the results of studies 2 and 3 give no evidence that desvenlafaxine inhibits CYP3A4 activity. In contrast, these findings suggest a decrease in the oral bioavailability of midazolam when administered with desvenlafaxine. Although reduced concentrations of midazolam would be consistent with induction of the CYP3A4 enzyme, reduction in concentrations of the CYP3A4 metabolite (1'-hydroxymidazolam) is not consistent with induction. In addition, both a pregnane $\mathrm{X}$ receptor/CYP3A4 promoter gene assay and tests for induction in primary cultures of human hepatocytes showed no evidence of CYP3A4 induction due to desvenlafaxine [2]. Therefore, induction is not a likely mechanism for the observed changes in midazolam pharmacokinetics. The exact mechanism for differences in absorption cannot be determined by the pharmacokinetic data generated from these studies. 
Citation: Nichols A, Liang Y, Matschke K, Paul J, Behrle J, et al. (2013) An Evaluation of the Potential of Cytochrome P450 3A4-Mediated Drug-Drug Interactions with Desvenlafaxine Use. J Bioequiv Availab 5: 053-059. doi:10.4172/jbb.1000134

\begin{tabular}{|l|l|l|l|l|}
\hline & Midazolam $\mathbf{n = 2 6}$ & Desvenlafaxine Titration $\mathbf{n = 2 5}$ & Desvenlafaxine + idazolam $\mathbf{n = 2 4}$ \\
\hline Any adverse event, $\mathrm{n}(\%)$ & $4(15.4)$ & $12(48.0)$ & $2(8.3)$ & Desvenlafaxine Taper $\mathbf{n = 2 4}$ \\
\hline Urinary hesitation & $2(7.7)$ & 0 & 0 & 0 \\
\hline Constipation & $1(3.8)$ & $3(12.0)$ & $1(4.2)$ & $1(4.2)$ \\
\hline Headache & $1(3.8)$ & $3(12.0)$ & 0 & 0 \\
\hline Asthenia & 0 & $3(12.0)$ & 0 & 0 \\
\hline Diarrhea & 0 & $2(8.0)$ & 0 & 0 \\
\hline Dizziness & 0 & $2(8.0)$ & 0 & 0 \\
\hline Dry mouth & 0 & $2(8.0)$ & 0 & 0 \\
\hline Nausea & 0 & $2(8.0)$ & 0 \\
\hline Somnolence & 0 & $2(8.0)$ & 0 \\
\hline Trismus & 0 & $2(8.0)$ & 0 \\
\hline
\end{tabular}

Table 5: Treatment-emergent adverse events observed in $\geq 2$ subjects during study 2 .

\begin{tabular}{|c|c|c|c|}
\hline & Midazolam $\mathrm{n}=\mathbf{2 8}$ & Desvenlafaxine $50 \mathrm{mg} \mathrm{n}=28$ & Desvenlafaxine + Midazolam $\mathbf{n}=\mathbf{2 8}$ \\
\hline Any adverse event, $\mathrm{n}(\%)$ & $21(75.0)$ & $16(57.1)$ & $24(85.7)$ \\
\hline Somnolence & $20(71.4)$ & 0 & $20(71.4)$ \\
\hline Abdominal pain & $1(3.6)$ & $4(14.3)$ & 0 \\
\hline Decreased appetite & 0 & $2(7.1)$ & 0 \\
\hline Diarrhea & 0 & $2(7.1)$ & 0 \\
\hline Fatigue & 0 & $3(10.7)$ & $4(14.3)$ \\
\hline Feeling abnormal & 0 & $2(7.1)$ & 0 \\
\hline Headache & 0 & $6(21.4)$ & $1(3.6)$ \\
\hline Insomnia & 0 & $3(10.7)$ & $1(3.6)$ \\
\hline Nausea & 0 & $3(10.7)$ & 0 \\
\hline
\end{tabular}

Table 6: Treatment-emergent adverse events observed in $\geq 2$ subjects during study 3 .

AEs in all 3 studies were generally mild to moderate, and the severity of TEAEs did not increase with concomitant administration. No serious AEs or discontinuations due to safety issues occurred in any of the studies.

\section{Limitations}

The findings of the current studies may be limited by the fact that all participants were healthy; therefore, these results may not be generalized to a patient population using multiple medications, particularly for major depressive disorder. In addition, the majority of subjects in these 3 studies were men. Body weight is a major source of differences in the pharmacokinetics of desvenlafaxine between men and women, however, and it is likely that similar results would be obtained in women, after adjustment for body weight.

\section{Conclusions}

Drugs that inhibit CYP3A4 may have a minor impact on the pharmacokinetics of desvenlafaxine. A minor decrease in exposure to drugs undergoing CYP3A4-mediated metabolism may occur after concomitant administration with desvenlafaxine. However, there was no indication that desvenlafaxine inhibited the metabolism of midazolam to 1'-hydroxymidazolam, which is primarily a CYP3A4mediated pathway. Based on these 3 studies, desvenlafaxine is not extensively metabolized by, nor does it measurably alter, CYP3A4mediated metabolism.

\section{Acknowledgments}

These studies were sponsored by Wyeth Research Inc., which was acquired by Pfizer in October 2009. Medical writing support was provided by Kathleen Dorries, PhD, of Peloton Advantage, LLC, and was funded by Pfizer Inc. No author received an honorarium or other form of financial support related to the development of this manuscript. The authors would like to acknowledge Nicolas Fauchoux, MD, Biotrial Rennes; Bryan G. Laura, MD, St. Mary's Physicians' Health Group; and Hetal S. Kocinsky, MD, Yale School of Medicine for their contributions as Principle Investigators for studies 1, 2, and 3, respectively.

\section{References}

1. Pristiq (2011) Philadelphia, PA: Wyeth Pharmaceuticals, Inc, a subsidiary of Pfizer Inc.

2. DeMaio W, Kane CP, Nichols Al, Jordan R (2011) Metabolism studies of desvenlafaxine. J Bioequiv Availab 3: 151-160.

3. Huang SM, Temple R, Throckmorton DC, Lesko LJ (2007) Drug interaction studies: study design, data analysis, and implications for dosing and labeling. Clin Pharmacol Ther 81: 298-304.

4. Ziegler WH, Schalch E, Leishman B, Eckert M (1983) Comparison of the effects of intravenously administered midazolam, triazolam and their hydroxy metabolites. Br J Clin Pharmacol 16 Suppl 1: 63S-69S

5. Gorski JC, Hall SD, Jones DR, VandenBranden M, Wrighton SA (1994) Regioselective biotransformation of midazolam by members of the human cytochrome P450 3A (CYP3A) subfamily. Biochem Pharmacol 47: 1643-1653.

6. Huang SM, Strong JM, Zhang L, Reynolds KS, Nallani S, et al. (2008) New era in drug interaction evaluation: US Food and Drug Administration update on CYP enzymes, transporters, and the guidance process. J Clin Pharmacol 48: 662-670.

7. Guidance for industry: bioavailability and bioequivalence studies for orally administered drug products - general considerations (2003) Silver Spring, MD: Food and Drug Administration.

8. DeVane CL, Donovan JL, Liston HL, Markowitz JS, Cheng KT, et al. (2004) Comparative CYP3A4 inhibitory effects of venlafaxine, fluoxetine, sertraline and nefazodone in healthy volunteers. J Clin Psychopharmacol 24: 4-10.

9. Hall J, Naranjo CA, Sproule BA, Herrmann N (2003) Pharmacokinetic and pharmacodynamic evaluation of the inhibition of alprazolam by citalopram and fluoxetine. J Clin Psychopharmacol 23: 349-357. 